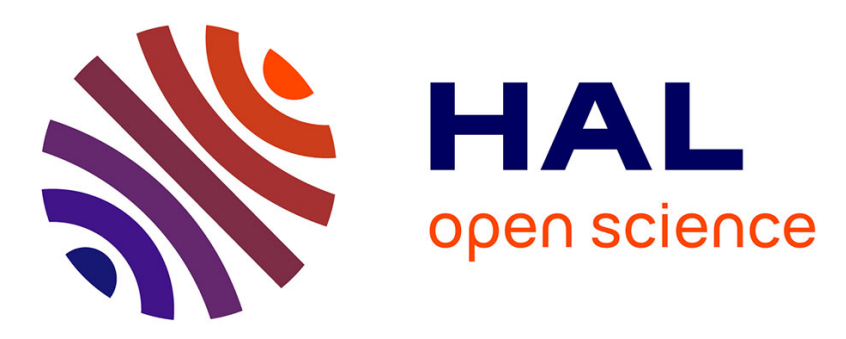

\title{
Analysis of flow separation using a local frame-axis: application to the open-channel bifurcation
}

Emmanuel Mignot, Delphine Doppler, Nicolas Riviere, Ivana Vinkovic, Jean-Noël Gence, Serge Simoëns

\section{- To cite this version:}

Emmanuel Mignot, Delphine Doppler, Nicolas Riviere, Ivana Vinkovic, Jean-Noël Gence, et al.. Analysis of flow separation using a local frame-axis: application to the open-channel bifurcation. Journal of Hydraulic Engineering, 2014, 140, pp.280-290. 10.1061/(ASCE)HY.1943-7900.0000828 . hal01296870

\section{HAL Id: hal-01296870 \\ https://hal.science/hal-01296870}

Submitted on 22 Nov 2018

HAL is a multi-disciplinary open access archive for the deposit and dissemination of scientific research documents, whether they are published or not. The documents may come from teaching and research institutions in France or abroad, or from public or private research centers.
L'archive ouverte pluridisciplinaire HAL, est destinée au dépôt et à la diffusion de documents scientifiques de niveau recherche, publiés ou non, émanant des établissements d'enseignement et de recherche français ou étrangers, des laboratoires publics ou privés. 


\section{Analysis of flow separation using a local frame-axis: \\ 2 application to the open-channel bifurcation}

3 Emmanuel Mignot ${ }^{*}, L M F A, C N R S$-Université de Lyon, INSA de Lyon, Université

4 Claude Bernard Lyon 1, Ecole Centrale de Lyon

5 Delphine Doppler, LMFA, CNRS-Université de Lyon, INSA de Lyon, Université

6 Claude Bernard Lyon 1, Ecole Centrale de Lyon

7 Nicolas Riviere, LMFA, CNRS-Université de Lyon, INSA de Lyon, Université Claude

8 Bernard Lyon 1, Ecole Centrale de Lyon

9 Ivana Vinkovic, LMFA, CNRS-Université de Lyon, INSA de Lyon, Université Claude

10 Bernard Lyon 1, Ecole Centrale de Lyon

11 Jean-Noel Gence, LMFA, CNRS-Université de Lyon, INSA de Lyon, Université Claude

12 Bernard Lyon 1, Ecole Centrale de Lyon

13 Serge Simoens, LMFA, CNRS-Université de Lyon, INSA de Lyon, Université Claude

14 Bernard Lyon 1, Ecole Centrale de Lyon

$15 *$ E-mail: emmanuel.mignot@insa-lyon.fr

17 Abstract

18 The motivation for the present work comes from the fact that a few recent publications

19 describing separation flows report a Counter-gradient Diffusion Phenomenon (CDP)

20 along the separating streamline. This CDP is related to i) a change of sign of the

21 Reynolds shear stress near the separating point and ii) an opposite sign between the

22 Reynolds shear stress and the mean shear rate, leading to a negative eddy-viscosity

23 coefficient. Such CDP is only reported for configurations where the angle between the

24 separating streamline and the main flow is large (referred to as Group 1), while no CDP

25 was reported for low angle configurations (referred to as Group 2). All these flows were

26 analyzed using Cartesian or curvilinear frame-axes. The aim of the present paper is to

27 propose a more intuitive frame for analysis, namely the Serret-Frenet frame-axis based

28 on the local flow direction, for which the CDP disappears for Group 1 flow

29 configurations and which highly improves the characterization of the mixing layer.

30 The recirculation zone occurring in the lateral branch of an open-channel bifurcation is

31 chosen as a separating flow configuration belonging to Group 1 and measured using

32 PIV. The characteristics of the mean flow and of the horizontal Reynolds stress are first

33 analyzed using a Cartesian frame-axis, in order to retrieve the CDP, which extension is

34 enhanced compared to the literature cases. Then the local Serret-Frenet frame-axis is

35 introduced and used for a second analysis of the data. The flow characteristics at the 
36 interface between the main flow and the recirculation zone accurately match the 37 characteristics of the well documented mixing layers available in the literature.

38 Moreover, the eddy-viscosity concept nicely applies using this Serret-Frenet frame-axis.

39 A simple geometrical analysis is finally performed in order to confirm that the negative

40 eddy-viscosity coefficient obtained when using a Cartesian frame-axis is only due to a

41 non-adequate frame of analysis.

\section{Introduction}

44 In the field or in laboratory conditions, a separating flow usually detaches from the 45 adjacent wall as it reaches a geometrical singularity. A recirculation zone (also named separating bubble) occurs along this wall. The typical velocity magnitude in the recirculation region is at least one order of magnitude lower than within the main flow and adverse streamwise velocities are observed near the wall. Meanwhile, the main flow accelerates as the main flow section is reduced. Further downstream, the main flow reattaches to the wall and recovers. Li and Djilali [1995] recall that the main parameters governing the flow separation are the Reynolds number of the flow and the geometry 52 itself.

53 In natural streams, separation zones in open channels have significant implications in 54 terms of sediment, gas or passive scalar (such as pollution or nutrients) exchanges 55 between the main flow which conveys material from upstream regions and the 56 recirculation zone where material is stored and is deposited if the density of the material exceeds that of water. Moreover, erosion may occur due to the acceleration of the main 58 flow near the maximum separation width location. Separation zones are also privileged zones of fish and plant reproduction and development. Interactions between the main

60 flow and the recirculation zone are thus of primary importance for bio-geo-chemical 61 processes and stream restoration.

62 In the literature, most attention was paid to the length of the recirculation as a function 63 of the characteristics of the main flow (Froude and Reynolds number, water depth, 64 discharge...) and of the geometry (angles, dimensions of the obstacle...). These studies 65 were dedicated to sudden lateral expansions [Babarutsi et al. 1989, Chu et al. 2004, 66 Riviere et al. 2011a], the lateral branch of $90^{\circ}$ open-channel bifurcations [Kasthuri and 67 Pundarikanthan 1987], the downstream branch of confluences [Best and Reid 1984, 68 Gurram et al. 1997] or forward-facing [Sherry et al. 2010] and backward-facing steps 69 [Adams and Johnston 1988]. 
71 When zooming at the interface between the main flow and the recirculation zone, very

72 high velocity gradients can be observed. In the upstream region, the separation zone can even be seen as a region of water almost at rest, entrained by the main flow. Large-scale coherent structures and high level turbulent intensities are present at the interface between the main flow and the recirculation zone, enhancing momentum and mass transfer. The transverse velocity gradient at the interface gives birth to a vertical mixing layer. A mixing layer is defined as "a turbulent flow that forms between two uniform, nearly parallel streams of different velocity" [Pope, 2000]. In the literature, most mixing layers have been studied in simple configurations such as:

80 - straight channels [Wygnanski \& Fiedler 1970; Bell \& Mehta 1990; Uijttewaal \& Booij 81 2000; Loucks \& Wallace 2012] or accelerated straight channels [Fieldler et al., 1991]. 82 Such analysis was performed using a Cartesian frame-axis $(x, y)$ with $x$ the axis of the 83 side walls and of the main flow and $y$ the transverse direction.

84 - curved channels [Margolis \& Lumley, 1965; Gibson \& Younis, 1983; Plesniak et al., 1996] analyzed using a curvilinear frame-axis $(r, \alpha)$ with $r$ the local distance to the center of curvature and $\alpha$ the angle formed by the radius at the source and the local 87 radius.

88 A review of these mixing layer characteristics was proposed by Mignot et al. (2013) 89 detailing the geometrical features and the mean and turbulent velocity distribution across the mixing layer. These studies revealed that the width of the mixing layer increases from up-to downstream and that the maximum of the Reynolds stress tensor components across the mixing layer occurs at its centerline.

\section{CDP phenomenon in mixing layers of separating flows}

95 Recent works were devoted to the analysis of the mixing layers in separating flows, 96 assuming that the separating streamline is the centerline of the mixing layer. These works confirmed that a maximum transverse gradient of streamwise velocity and a maximum value of all components in the Reynolds stress tensor are measured along the

99 separating streamline.

100 The so-called "CDP phenomenon" was reported by some author in such separation

101 flows. This phenomenon is based on the eddy viscosity concept, following the works of 102 Joseph Boussinesq in 1877. It relates the Reynolds stress tensor to the mean rate of strain tensor and writes: 
$104-\overline{u_{i}{ }^{\prime} u_{j}{ }^{\prime}}+\frac{2}{3} k \delta_{i j}=v_{T}^{C}\left(\frac{\partial \overline{u_{i}}}{\partial x_{j}}+\frac{\partial \overline{u_{j}}}{\partial x_{i}}\right)=2 v_{T}^{C} S_{i j}$

105 where $k$ is the turbulent kinetic energy, $v_{T}^{C}$ is the so-called "turbulent viscosity" or 106 "eddy viscosity" coefficient in a Cartesian frame-axis and $S_{\mathrm{ij}}$ is the mean rate of strain

107 tensor (with $u=\bar{u}+u^{\prime}$ the instantaneous fluid velocity, where the over-bar denotes 108 Reynolds-averaging - or time-averaging - and prime denotes fluctuation). In Eq. (1), $v_{T}^{C}$

109 is hypothesized isotropic as it does not depend on $i$ and $j$. Nevertheless, it is well known 110 that this hypothesis of isotropy is not satisfied in many configurations [see Pope, 2000], 111 such as in flows with significant streamline curvature due to "the subtle way curvature 112 influences the mean flow" [Patel and Sotiropoulos, 1997]. We do not expect Eq. (1) to 113 be valid in the present flow configuration and no discussion regarding the validity of the 114 isotropic turbulent viscosity assumption is made here.

115 In a 2D analysis, application of Eq. (1) to the non-diagonal term writes: $-\overline{u^{\prime} v^{\prime}}=v_{T}^{C}\left(\frac{\partial \bar{u}}{\partial y}+\frac{\partial \bar{v}}{\partial x}\right)=2 v_{T}^{C} S_{x y}$

117 with $x$ and $y$ the streamwise and transverse axes respectively, $u$ and $v$ the instantaneous 118 velocity components along $x$ and $y$ respectively and $S_{x y}$ the mean shear rate.

119 If, locally, the sign of the Reynolds shear stress $-\overline{u^{\prime} v^{\prime}}$ and of the mean shear rate $S_{\mathrm{xy}}$ are 120 opposite, $v_{T}^{C}$ becomes negative at this location and the so-called CDP (for Counter121 gradient Diffusion Phenomenon, see Hattori and Nagano [2010]) takes place. The 122 negative eddy viscosity coefficient (and thus the CDP) near the separating point was 123 implied by Simoens et al. [2007] data in a flow separating over a square-shape obstacle, 124 over a forward-facing step by Largeau and Moriniere [2007] and later by Sherry et al. 125 [2010] and Ren and Wu [2011] and was computed using DNS by Hattori and Nagano 126 [2010]. Further downstream, the Reynolds shear stress profiles become positive 127 throughout the flow and the eddy viscosity coefficient becomes positive. Similarly, 128 within the flow separating at the inner bank of a sharp bend, Blanckaert et al [2013] 129 observed a similar CDP region by explicitly reporting an opposite sign between gradient 130 of mean velocity and Reynolds shear stress in curvilinear frame-axis. These three 131 configurations (sharp angle obstacle - forward facing step - and sharp bend) obviously 132 experience the same phenomenon leading to CDP (which will be explained further 133 below) and are referred to as "Group 1" in the present paper. Such CDP was also 
134 observed in turbulent scalar transport experiments where the gradient of mean 135 concentration (or temperature) and the corresponding flux can be of same sign locally 136 [see Paranthoen et al., 2004]. Separating flows downstream groynes and in the outlet 137 branch of confluences and bifurcations (as in the present configuration) should be added 138 to this list even though no evidence of the CDP in these geometries could be found in 139 the literature. The specificity of these flows is that, at the separation point, the angle 140 between the separating streamline $(S s)$ and the main axis $(x)$ is large (this angle is noted $141 \theta$, see Fig. 1).

142 Oppositely, to the authors knowledge none of the experiments and calculations related 143 to separating flows in downward-facing steps - downstream from hills - in wakes or in 144 sudden lateral expansion reported this CDP phenomenon: In these studies referred to as 145 "Group 2", the Reynolds shear stress $-\overline{u^{\prime} v^{\prime}}$ and mean shear rate $S_{\mathrm{xy}}$ remain of same

146 sign from the separation point towards downstream and $v_{T}^{C}$ thus remains positive. The 147 recirculation length is equal to 6-8 times the expansion (or step) width, except in very 148 shallow conditions [see Chu et al., 2004 or Adams and Johnston, 1988]. Due to such 149 elongated shape, the direction of the separating streamline $\left(s_{s}\right)$ is quite parallel to the 150 main axis $(x)$ until approaching the reattachment point where the flow pattern becomes 151 much more complex [see Riviere et al. 2011a].

152 The difference between groups 1 and 2 can thus be explained based on geometrical aspects related to the magnitude of the $\theta$ angle.

\section{Objectives}

156 In the present paper we aim at showing that mixing layers in Groups 1 and 2 are 157 actually similar as long as a proper frame-axis is used for the data analysis. We propose 158 a new methodology using the local frame-axis for the analysis of separating flows. Main 159 advantages are thus that there is i) no change of sign of the Reynolds shear stress, ii) no $160 \mathrm{CDP}$ and iii) direct access to the mixing layer characteristics. A laboratory bifurcation 161 flow (belonging to Group 1) is chosen for analysis. After describing the experimental 162 set-up used to measure the bifurcation flow pattern, the local frame-axis is introduced. 163 The mean and turbulent flow characteristics are then computed and the Reynolds shear 164 stress obtained using the Cartesian and local frame-axis are compared in order to 165 discuss the advantages of the local frame-axis. Last section is finally devoted to reveal that CDP is only related to geometrical features. 


\section{Experimental set-up}

169 The open-channel bifurcation flow field is measured in the channel intersection facility 170 at the Laboratoire de Mécanique des Fluides et d'Acoustique (LMFA) at the University 171 of Lyon (INSA-Lyon, France) sketched in Fig. 2 and adapted from [Riviere et al. 172 2011b]. The facility consists of three horizontal glass channels of $b=0.3 \mathrm{~m}$ width each, $173 L_{\mathrm{u}}=2 \mathrm{~m}$ length for the upstream branch and $L_{\mathrm{b}}=L_{\mathrm{d}}=2.6 \mathrm{~m}$ for both outlet branches. The 174 channels intersect at $90^{\circ}$ with the "upstream branch" along $y$ axis, the "lateral branch" 175 along $x$ axis and the "downstream branch" along $y$ axis, that is aligned with the upstream branch. The upstream branch is connected to a large storage tank. When leaving the upstream tank, the water passes through a honeycomb that stabilizes and straightens the flow. This ensures quasi-1D flows within the incoming channels, even though fully developed inflow conditions would require a considerably longer upstream channel. When reaching the bifurcation, the inflow separates, flows through each outlet branches and is collected by the outlet tanks. The three parameters which govern the flow configuration are: the upstream flow rate $Q_{u}=4 \mathrm{~L} / \mathrm{s}$ and the water depth at the downstream end of the lateral $\left(h_{b}=12.0 \mathrm{~cm}\right)$ and downstream branches $\left(h_{d}=12.1 \mathrm{~cm}\right)$, which are controlled by sharp crested weirs (noted $C_{b}$ and $C_{d}$ in Fig. 2). In the inlet branch when approaching the bifurcation the water depth equals $h_{u}=12.1 \mathrm{~cm}$, leading to an upstream Reynolds number $R_{\mathrm{u}}=30000$, based on the mean velocity and hydraulic diameter. Given the limited measured water depth decrease from the upstream $\left(h_{u}\right)$ to the lateral $\left(h_{b}\right)$ branch, leading to a water depth decrease of about $1 \mathrm{~mm}$ over a length of $2 \mathrm{~m}$ in the lateral branch, water depth changes are assumed to be negligible. The lateral tank is connected to the downstream tank and the water is pumped from the downstream to the upstream tank. The upstream and lateral flow-rates are measured in the pumping loops using electromagnetic flow meters $(+/-0.05 \mathrm{~L} / \mathrm{s})$, see Fig. 2 . The values of the discharges measured in the lateral and downstream branches are similar $Q_{b}=Q_{d}=2 \mathrm{~L} / \mathrm{s}$

195 Velocity fields are measured using a horizontal 2D-PIV technique. A slide projector 196 along with a diaphragm is used to create a collimated $5 \mathrm{~mm}$ thick light sheet at the measured elevation in the channel intersection. A 1280x960 pixel progressive CCDcamera mounted with a $8 \mathrm{~mm}$ focal lens is connected to a PC computer through a

199 Firewire acquisition card. It is placed at an elevation of about $1.5 \mathrm{~m}$ above the free 200 surface. Polyamid particles (50 $\mu \mathrm{m}$ diameter) are added to the water and act as tracers. 
201 Inserting the whole set-up in the dark finally permits to record the tracer motion at the

202 lightened elevation at a fixed frame-rate of $30 \mathrm{~Hz}$ during $133 \mathrm{~s}$ with 8 bit grey-levels on a $203400 \mathrm{~mm} \times 300 \mathrm{~mm}$ large window with a horizontal resolution of $0.5 \mathrm{~mm}$ per pixel. The 204 PIV commercial software Davis (from Lavision) permits to correct the optical 205 distortions, to subtract the background and to compute the velocity field. The final 206 velocity uncertainty is estimated to $1.5 \mathrm{~mm} / \mathrm{s}(0.1 *$ spatial resolution $[0.5 \mathrm{~mm} / \mathrm{pixel}]$ * 207 acquisition frequency [30 Hz]). The PIV system provides the two horizontal velocity 208 components $\mathrm{u}$ and $\mathrm{v}$ at each location of the measurement grid and at each time step with 209 high spatial resolution without any intrusion. Erroneous vectors are detected by i) too 210 weak correlation between two consecutive images, ii) presence of two or more peaks of 211 correlations of similar correlation coefficients or iii) local velocity strongly differing 212 from the neighboring locations of the grid and are not considered in the time-averaged 213 statistics (mean velocity or any term of the Reynolds stress tensor) at this location.

214 Two neighboring measurement zones are presented in the sequel, which frontier is 215 located at $x=400 \mathrm{~mm}$ (see Fig. 3). The final data used in the following, is a grid of points 216 within the bifurcation and the lateral branch composed each of 4000 sample long (133s) $217 u$ (along $x$ axis) and $v$ (along $y$ axis) velocity signals. Measurements were taken at 218 elevations $z=4 \mathrm{~cm}$ and $9 \mathrm{~cm}$. Changes in the flow features are expected when 219 approaching the bed, but limited differences were observed between data at both 220 elevations, and only data at $9 \mathrm{~cm}$ are presented herein.

\section{Mean flow}

223 The mean velocity field, averaged over 4000 samples is shown on Fig. 3. As described 224 by Neary et al. [1999], as the main flow reaches the intersection, one part reaches the 225 downstream branch and the other part is deflected towards the lateral branch. The flow 226 in the lateral branch detaches at the upstream corner $(x=300 \mathrm{~mm} ; y=0)$ and the lateral 227 main flow is confined very near its left bank (y 300mm). A zoom in the recirculating 228 region (in Fig. 3) reveals that its core takes place at $x \sim 650 \mathrm{~mm}$ and $y \sim 150 \mathrm{~mm}$. In the 229 region between the core and the right bank, the mean flow is oriented towards the 230 intersection, with a velocity magnitude one order of magnitude lower than the velocity 231 magnitude of the main flow. The so-called "separating streamline" is defined as the 232 streamline starting at the upstream corner of the intersection (see Fig. 3). This 233 streamline is constructed using the following steps: i) the local velocity is interpolated 234 from the measured mean velocity field shown in Fig. 3 which permits to calculate the 
235 local flow direction, ii) a small displacement of about one millimeter is computed along

236 this direction leading to a new location. Steps i) and ii) are then repeated until reaching the limit of the measured area. Locations computed at step ii) finally define the

238 streamline. In the upstream portion of the separating streamline, a very intense velocity 239 gradient occurs between the main and recirculation flow. Further downstream, the 240 velocity gradient across the separating streamline appears to decrease.

\section{Eddy viscosity concept using a Cartesian frame-axis $(x, y)$}

243 In the sequel, most attention will be paid to the vicinity of the separating streamline.

244 The distribution of mean shear rate $2 S_{x y}=\partial \bar{u} / \partial y+\partial \bar{v} / \partial x$ in the lateral branch is 245 depicted in Fig. 4. $2 S_{x y}$ is negative in the vicinity of the separating streamline in the 246 upstream region and positive elsewhere. In the downstream region of the lateral branch, 247 the maximum positive value of $2 S_{x y}$ is measured close to the separating streamline. Fig. 2484 also shows the distribution of Cartesian horizontal Reynolds shear stress $-\overline{u^{\prime} v^{\prime}}$. This 249 term is i) negative in the whole upstream region of the lateral branch $(x<450 \mathrm{~mm})$, ii) 250 positive in the downstream region $(x>650 \mathrm{~mm})$ and iii) both negative (near the left bank) 251 and positive (in the recirculation region) in the intermediate region. As discussed in the 252 introduction, the negative horizontal Reynolds shear stress in the upstream region of the 253 separation was reported in all Group 1 flow configurations. In the present Group 1 case, 254 the particularly high angle $\theta$ between the separating streamline and the main flow in the 255 lateral branch (equal to $85.1^{\circ}$ at the geometrical singularity: $x=300 \mathrm{~mm} \& y=0 \mathrm{~mm}$ ) 256 enhances the extension and magnitude of the negative Reynolds shear stress region.

257 Fig. 4 thus confirms that the mean shear rate $2 S_{x y}$ and Reynolds shear stress $-\overline{u^{\prime} v^{\prime}}$ have 258 opposite sign and that the flow experiences consequently a CDP with negative eddy 259 viscosity coefficient at two locations (shading in Fig. 4):

$260-$ In the upstream region $(\mathrm{x}<600 \mathrm{~mm})$, near the separating streamline and on the $261 \quad$ left bank side.

- Within the recirculation region at some non-analyzed locations.

264 The CDP in the present configuration is finally similar to all observed CDP in the 265 literature except it is certainly enhanced due to the high $\theta$ angle. The application of the 266 eddy viscosity concept in the Cartesian frame-axis (Eq. 2) is presented in Fig. 5. A bestqualitative constant eddy-viscosity coefficient value $v_{T}^{C}=8.10^{-5} \mathrm{~m}^{2} / \mathrm{s}$ is chosen by 
268 comparing Figs. 5a and 5b. The poor shape agreement between $-\overline{u^{\prime} v^{\prime}}$ and $2 v_{T}^{C} S_{x y}$ in 269 terms of signs and magnitudes in the vicinity of the separating streamline confirms the 270 poor applicability of the eddy-viscosity concept in the present separating flow 271 configuration except in the downstream region.

272 Nevertheless, it should be noted that, while the CDP leads to a negative $P_{M}=-\overline{u^{\prime} v^{\prime}} \cdot 2 S_{x y}$ 273 turbulent kinetic energy production locally (Durbin [1993] wrote that "The negative 274 shear stress causes negative energy production in this region and this reduces the 275 turbulent kinetic energy"), the sum of all turbulent production terms $276 P=-\overline{u_{i}^{\prime} u_{j}^{\prime}} \partial \overline{u_{i}} / \partial x_{j}$ remains positive (not shown here). Mixing layer analysis and eddy viscosity concept using a Serret-Frenet frame-axis Application of Serret-Frenet frame-axis

280 Another approach for analyzing the separating flow is proposed using a so-called 2D 281 Serret-Frenet (s, n) coordinate system (or local frame-axis) based on the direction of the mean velocity in each point. Fig. 6a shows i) the streamlines, ii) the so-called separating streamline, i.e. the streamline which initiates at the upstream corner and iii) the fieldlines which are perpendicular to the velocity field at each location. All fieldlines have an extremity at one of the banks and the other within the core of the recirculation. Here, the unit vector $\mathbf{s}$ is directed along the mean velocity (i.e. along the local streamline), $\mathbf{n}$ is perpendicular to $\mathbf{s}$, directed along the fieldline towards the center of curvature of the streamlines (see Fig. 6b). When projecting the velocity field to the local

289 axis system, it comes that $\overline{u_{s}}=\sqrt{\overline{u^{2}+\bar{v}^{2}}}$ and $\overline{u_{n}}=0$ but $u_{n}^{\prime} \neq 0$. Moreover, a global curvilinear coordinate system based on the separating streamline is set so that $(\mathbf{S}, \mathbf{N})$ is the particular Serret-Frenet axis- system attached to the separating streamline (thick line in Fig. 6b), $S$ denotes the distance from the upstream corner along the separating streamline and $N$ denotes the distance from this streamline along any fieldline (with $N=$ 0 on the separating streamline). Fig. $6 \mathrm{~b}$ shows that, when plotted in Cartesian frame, $\boldsymbol{n}$ and $N$ axes are directed towards the core of the recirculation region. In Fig. 6b, seven fieldlines crossing the separating streamline at different $S$ are depicted and are selected for presenting the data in the following figures. 
300 As exposed above, in the 2D Serret-Frenet frame-axis, only one mean velocity

301 component is not equal to zero, i.e. $\overline{u_{s}}$. Fig. 7a presents profiles of streamwise velocity

$302 \bar{u}_{s}$ along the 7 selected fieldlines plotted in Fig. 6b. The streamwise velocity always

303 decreases from the main flow towards the recirculation zone, that is for increasing $N$.

304 Fig. $7 \mathrm{~b}$ and $7 \mathrm{c}$ then show:

305 - That the normal gradient of streamwise velocity (Fig. 7b) and the Reynolds shear stress (Fig. 7c) are mainly negative. It is important to note that the negative signs of velocity gradient (Fig. 7b) and of Reynolds shear stress (Fig. 7c) are a consequence of the orientation of $\mathbf{n}$ towards the core of the recirculation region: if the orientation of $\mathbf{n}$ had been chosen in opposite direction (towards the left bank), both $\partial \overline{u_{s}} / \partial n$ and $\overline{-\bar{u}_{s}^{\prime} u_{n}^{\prime}}$ would have been positive.

- That the absolute normal gradient of streamwise velocity and absolute Reynolds shear stress are maximum close to the separating streamline for all $S$ and decrease on both sides. The small shift of maximum absolute of these two terms towards the recirculation zone $(N>0)$ may be related to the shift of the mixing layer towards the slower flow (see Figs. 6 and 7 of van Prooijen and Uijttewaal [2002]) or due to $3 \mathrm{D}$ effects and was observed for instance in Fig. 4 in [Uijttewaal and Booij, 2000] or in Figs. 5 and 9 in [Gibson and Younis, 1983].

- And that the absolute gradient and absolute Reynolds shear stress are maximum very close to the separating corner $(x \sim 300 \mathrm{~mm}$ and $y \sim 0)$ and slowly decrease towards downstream along $S$.

To define the mixing-layer, let us introduce the outer velocities $U 1(S)=\bar{u}\left(S, N_{1}\right)$ and $U 2(S)=\bar{u}\left(S, N_{2}\right)$ with $N_{1}<0$ and $N_{2}>0$, as the streamwise velocities located where $\partial \overline{u_{s}} / \partial n$ becomes negligible. Fig. 8a confirms that $U 2(S)$ is almost equal to zero while $U 1(S)$ remains quite constant at a magnitude of about $0.15 \mathrm{~m} . \mathrm{s}^{-1}$. As a consequence, the velocity difference $\Delta U(S)=U 1(S)-U 2(S)$ remains quite constant for all $S$ (Fig. 8b).

326 Following the approaches available in the literature, the width of the mixing layer $\delta(S)$

327 for all $S$ is defined as: 
331 Fig. 8c reveals that $\delta(S)$ increases for increasing $S$ (in agreement with mixing layers in 332 the literature as observed by Gibson and Younis [1983] or Bell and Mehta [1990]) and 333 reaches a plateau for $S>400 \mathrm{~mm}$ as the separating streamline becomes parallel to $x$ axis.

334 Saturation of the mixing layer width might be due to the confinement and its interaction 335 with the boundary layer on the lateral wall.

337 Using the velocity scale $\Delta U(S)$ and the spatial scale $\delta(S)$, Fig. 9 shows the non338 dimensional mean streamwise velocity profiles along the 7 fieldlines of Fig. $6 \mathrm{~b}$. It 339 appears that for $S>50 \mathrm{~mm}$, the velocity profiles become self-similar in the vicinity of the 340 separating streamline $(-0.5<N / \delta<1.25)$. This behavior is in agreement with straight 341 [Wygnanski \& Fiedler, 1970] and curved [Gibson \& Younis, 1983] mixing layers.

343 Figs. 7, 8 and 9 thus confirm the fair agreement of the present mixing layer analyzed 344 using a Serret-Frenet coordinate system with the more classical straight and curved 345 mixing layers analyzed using Cartesian and curvilinear coordinate systems respectively 346 in the literature.

\section{Eddy viscosity concept using a Serret-Frenet frame-axis}

349 It can be shown [see Mignot et al., 2013] that in the Serret-Frenet frame-axis, the 350 Reynolds tensor writes:

$$
\mathbf{R}_{S F}=\left[\begin{array}{cc}
\overline{-\overline{u_{s}^{\prime 2}}} & -\overline{u_{s}^{\prime} u_{n}^{\prime}} \\
-\overline{u_{s}^{\prime} u_{n}^{\prime}} & -\overline{u_{n}^{\prime 2}}
\end{array}\right]
$$

352 And the mean rate of strain tensor writes:

$$
\mathbf{S}_{S F}=\left[\begin{array}{cc}
\frac{\partial \overline{u_{s}}}{\partial s} & \frac{1}{2}\left(\frac{\partial \overline{u_{s}}}{\partial n}+\frac{\overline{u_{s}}}{R_{s}}\right) \\
\frac{1}{2}\left(\frac{\partial \bar{u}_{s}}{\partial n}+\frac{\overline{u_{s}}}{R_{s}}\right) & \frac{\overline{u_{s}}}{R_{n}}
\end{array}\right]
$$

354 Where $R_{s}$ and $R_{n}$ are the local radius of curvature of the streamlines and fieldlines, 355 respectively. By analogy with the Eq. 2, we introduce the eddy-viscosity concept in the 356 Serret-Frenet frame-axis using the non-diagonal terms of $\mathbf{R}_{\mathrm{SF}}$ and $\mathbf{S}_{\mathrm{SF}}$ (Eqs. 4-5) as:

$$
-\overline{u_{s}^{\prime} u_{n}^{\prime}}=v_{T}^{S F}\left(\frac{\partial \overline{u_{s}}}{\partial n}+\frac{\overline{u_{s}}}{R_{s}}\right)=v_{T}^{S F} \cdot 2 S_{s n}
$$


360 Fig. 10a presents the distribution of the horizontal component of Reynolds shear stress

361 in the Serret-Frenet frame-axis $-\overline{u_{s}^{\prime} u_{n}^{\prime}}$ within the lateral branch of the bifurcation

362 (with the same data as in Fig. 7c). Unlike the analysis using a Cartesian frame-axis (see

363 previous sections and Fig. 5), no change of sign of $-\overline{u_{s}^{\prime} u_{n}^{\prime}}$ is observed over the whole 364 measured region.

365 Fig. 10b shows the spatial evolution of $2 v_{T}^{S F} S_{s n}$, where the value of the constant eddy366 viscosity coefficient $v_{T}^{S F}=5.10^{-5} \mathrm{~m}^{2} / \mathrm{s}$ is chosen by comparing Figs. 10a and 10b. The 367 fair agreement between these two graphs confirms the high degree of application of the 368 eddy-viscosity concept using the Serret-Frenet frame-axis. Most importantly, unlike the 369 Cartesian frame-axis, no region of opposite signs between $-\overline{u_{s}^{\prime} u_{n}^{\prime}}$ and $370 v_{T}^{S F}\left(\partial \overline{u_{s}} / \partial n+\overline{u_{s}} / R_{s}\right)$ - that is no CDP region - is observed along the separating 371 streamline when using the Serret-Frenet frame-axis. However a limited region of 372 positive, but limited, $v_{T}^{S F}\left(\partial \overline{u_{s}} / \partial n+\overline{u_{s}} / R_{s}\right)$ values takes place as the main flow rotates 373 when entering the lateral branch $(x \sim 350 \mathrm{~mm}$ and $y \sim 250 \mathrm{~mm})$, near the upstream top 374 corner $(x=y=300 \mathrm{~mm})$ of the intersection. The failure of applicability of Eq. (6) in this 375 area may be related to the complex flow near the corner where a bow wave and a 376 horseshoe vortex take place and thus where 3D aspects of the flow should not be negligible. Application of the eddy-viscosity concept in this area would then require 3D

378 flow data, which is not available using the present 2D-PIV experimental approach.

380 In order to analyze the spatial variations along $S$ of the eddy-viscosity coefficient, $v_{T}^{S F}$ 381 was computed using two approaches along each of the seven fieldlines of Fig. 6b: square fitting method over each fieldline in the vicinity of the mixing layer (for $60 \mathrm{~mm}<N<100 \mathrm{~mm})$.

- by applying Prandtl mixing length model using the empirical relation between the mixing length and the mixing layer width initially introduced by Kuethe [1935] for a jet and summarized in Rodi [1993]: $v_{T-\text { Prandtl }}^{\mathrm{SF}}=(0.07 \delta)^{2}\left|\partial \overline{u_{s}} / \partial n+\overline{u_{s}} / R_{s}\right|$ where the absolute value in this expression 
is evaluated for each fieldline at the location of maximum streamwise velocity gradient $\partial \overline{u_{s}} / \partial n$.

391 Fig. 11 shows that these two eddy-viscosity coefficients tend to increase along $S$ from the separation point towards downstream. Both estimates give quite similar results: about $2.10^{-5}$ to $6.10^{-5} \mathrm{~m}^{2} / \mathrm{s}$, confirming the value $v_{T}^{S F}=5.10^{-5} \mathrm{~m}^{2} / \mathrm{s}$ obtained as a qualitative constant determination over the whole region (Fig. 10). This validates the use of Prandtl mixing length theory for separating mixing layers even though the Prandtl mixing length estimated coefficient remains lower by $5 \%$ to $50 \%$ compared to the best-fit coefficient along the mixing layer.

In order to calculate the error made when estimating the Reynolds shear stress, the eddy-viscosity concept (Eq. 6) is applied using these eddy-viscosity coefficients in Fig. 12 along the seven fieldlines shown on Fig. 6b. Fig. 12 thus compares:

402 - the measured Reynolds shear stress $-\overline{u_{s}^{\prime} u_{n}^{\prime}}$,

$403-v_{T-\text { Best Fit }}^{S F}\left(\partial \overline{u_{s}} / \partial n+\overline{u_{s}} / R_{s}\right)$

$404-v_{T-\text { Best Fit }}^{S F}\left(\partial \overline{u_{s}} / \partial n\right)$ which is a simplified version of Eq. (6) considering only the term

405 involving the streamwise velocity gradient and neglecting the radius of curvature.

$406-v_{T-\text { Prandtl }}^{\mathrm{SF}}\left(\partial \overline{u_{s}} / \partial n+\overline{u_{s}} / R_{s}\right)$.

407 This figure reveals that the eddy-viscosity concept (Eq. 6) considering the best-fit 408 coefficient $v_{T-\text { Best Fit }}^{S F}$ along each fieldline (diamonds) fairly predicts the Reynolds shear 409 stress. On the other hand, the simplified eddy-viscosity concept, considering $v_{T \text {-Best Fit }}^{S F}$ 410 (circles) leads to a very similar estimate of the Reynolds shear stress as Eq. (6). This 411 confirms that the curvature effect can be neglected here with regards to the streamwise 412 velocity gradient along $N$. Finally, the eddy-viscosity concept (Eq. 6) considering an 413 eddy-viscosity coefficient obtained through the Prandtl mixing length approach $v_{T \text {-Prandtl }}^{\mathrm{SF}}$ 414 (full squares) results in a fair shape of the Reynolds shear stress but strongly 415 underestimates its magnitude (as could be predicted from Fig. 11).

\section{Geometrical analysis}


418 In the previous sections, we showed that the eddy viscosity concept in the Cartesian

419 frame-axis (Eq. 2) is not relevant as the eddy viscosity coefficient $v_{T}^{C}$ would

420 dramatically vary from one location to another and would reach negative values in the

421 near-separation region for Group 1 with high $\theta$ values. Oppositely, we showed that the

422 eddy viscosity concept in the Serret-Frenet frame-axis (Eq. 6) is relevant in the whole

423 domain and leads to a quasi-constant eddy viscosity coefficient $v_{T}^{S F}=5.10^{-5} \mathrm{~m}^{2} / \mathrm{s}$ (see

424 Fig. 10). This is coherent with Boussinesq's idea that applies for uniform shear flows in

425 the direction of maximum shear. The separating flow can thus be considered as an

426 almost uniform shear flow in the local frame-axis, while it is not the case in the

427 Cartesian frame axis. Once accepted that the eddy viscosity concept applies in the

428 Serret-Frenet frame axis, occurrence of negative $v_{T}^{C}$ coefficients for high $\theta$ values can

429 be explained using geometrical considerations as exposed below and in the appendix.

430 Consider the local Cartesian frame-axis $(x, y)$ and the Serret-Frenet frame-axis $(s, n)$ as

431 depicted in Fig. 13.

432

433 The projection of the Serret-Frenet axis (SF) in the Cartesian (C) axis writes:

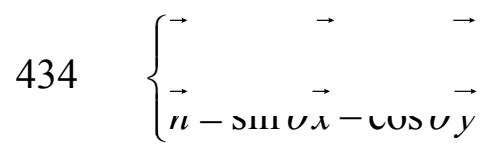

435 The rotation matrix $P_{C}^{S F}$ that allows frame change from $\mathrm{C}$ to $\mathrm{SF}$ (and its counterpart $\left.436 P_{S F}^{C}\right)$ thus writes:

$437 \quad P_{C}^{S F}=\left[\begin{array}{cc}\cos \theta & \sin \theta \\ \sin \theta & -\cos \theta\end{array}\right]$ and $P_{S F}^{C}=P_{C}^{S F^{-1}}=\left[\begin{array}{cc}\cos \theta & \sin \theta \\ \sin \theta & -\cos \theta\end{array}\right]$

438 As a consequence, the relationship between the components of the instantaneous

439 velocity vector $\vec{v}-\overrightarrow{u x}+v \vec{y}-u_{s} \vec{s}+u_{n} \vec{\imath}$ is straightforward:

$440 \quad\left[\begin{array}{l}u \\ v\end{array}\right]_{C}=P_{C}^{S F}\left[\begin{array}{l}u_{s} \\ u_{n}\end{array}\right]_{S F}=\left[\begin{array}{cc}\cos \theta & \sin \theta \\ \sin \theta & -\cos \theta\end{array}\right]\left[\begin{array}{l}u_{s} \\ u_{n}\end{array}\right]_{S F}$

441 Then, the Reynolds stress tensor $\mathbf{R}_{S F}$ (Eq. 4) and the mean rate of strain tensor $\mathbf{S}_{S F}$ (Eq.

442 5) in the Serret-Frenet frame can be expressed as a function of the Reynolds stress 443 tensor $\mathbf{R}_{C}=-\overline{u_{i}^{\prime} u_{j}^{\prime}}$ and the mean rate of strain tensor $\mathbf{S}_{C}=\left(\partial \overline{u_{i}} / \partial x_{j}+\partial \overline{u_{j}} / \partial x_{i}\right) / 2$ in Cartesian 444 frame as:

$445 \quad \mathbf{R}_{S F}=P_{S F}^{C} \mathbf{R}_{C} P_{C}^{S F}$ and $\mathbf{S}_{S F}=P_{S F}^{C} \mathbf{S}_{C} P_{C}^{S F}$ 
446 As detailed in the appendix, i) applying Eq. (10) to $\mathbf{R}_{x y}$ (defined in Eq. 11) and $\mathbf{S}_{x y}$

447 (defined in Eq. 12), ii) applying the eddy viscosity concept in the Cartesian frame-axis 448 (Eq. 2) and iii) including the eddy viscosity concept in the Serret-Frenet frame-axis (Eq.

449 6) finally leads to Eq. (15):

450

$$
v_{T}^{C}=\frac{-v_{T}^{S F}\left(\frac{\partial \overline{u_{s}}}{\partial n}+\frac{\overline{u_{s}}}{R_{s}}\right)+\tan (2 \theta)\left(\overline{u_{n}^{\prime 2}}-\overline{u_{s}^{\prime 2}}\right) / 2}{-\left(\frac{\partial \overline{u_{s}}}{\partial n}+\frac{\overline{u_{s}}}{R_{s}}\right)+\tan (2 \theta)\left(\frac{\partial \overline{u_{s}}}{\partial s}-\frac{\overline{u_{s}}}{R_{n}}\right)}
$$

451 We have shown that $v_{T}^{S F}$ remains positive except near the upstream top corner of the 452 intersection where the 2D eddy-viscosity concept does not apply (see Fig. 10). The sign 453 of $v_{T}^{C}$ thus depends on $\theta$ :

- For low $\theta, \tan (2 \theta)$ is small so that: i) the denominator is dominated by the first term (note that $\partial \overline{u_{s}} / \partial n \gg \quad$ in the mixing layer, see Fig. 7a) and ii) as tangential and longitudinal velocity variances have similar orders of magnitude in the present data (not shown here) and in the literature (see for instance Gibson et al. [1983]'s data), the numerator is also dominated by the first term. As a consequence, $v_{T}^{C}>0$ and $\lim _{\theta \rightarrow 0} v_{T}^{C}=v_{T}^{S F}$.

- For $\theta$ approaching $\pi / 4, \tan (2 \theta)$ becomes infinite and $\lim _{\theta \rightarrow \pi / 4} v_{T}^{C}=\frac{1}{2} \frac{\overline{u_{s}^{\prime 2}}-\overline{u_{n}^{\prime 2}}}{-\frac{\partial \overline{u_{s}}}{\partial s}+\frac{\overline{u_{s}}}{R_{n}}}$ However, $\overline{u_{s}^{\prime 2}}>\overline{u_{n}^{\prime 2}}$ in the present experiment (not shown here) as in Cartesian frame-axis in the literature (see for instance Gibson et al. [1983] or Bell and Mehta [1990]'s data) so that the numerator is positive. Moreover, in the regions where $\theta \sim \pi / 4$, especially near the separating streamline $(100 \mathrm{~mm}<\mathrm{S}<300 \mathrm{~mm})$, the denominator is negative (not shown here) leading to a negative $v_{T}^{C}$ and thus to the may take negative values while $v_{T}^{S F}$ remains positive. The apparent counter-gradient diffusion phenomenon is simply due to the fact that the eddy viscosity concept applies only in the direction of maximum shear, which is the flow direction $(s)$. This fact also explains that, when using Serret-Frenet frame instead of Cartesian frame, better agreement is achieved between the present experimental velocity profiles or Reynolds 
473 stress profiles and classical mixing layer profiles.

474 At last, for flows of Group 2, the direction of the separating streamline $(S)$ is quite 475 parallel to the main axis ( $x$ ) until approaching the reattachment point where the flow 476 pattern becomes much more complex [see Riviere et al. 2011]. As regards to the present

477 analysis, since $\theta$ remains low, $v_{T}^{C}$ remains positive, approaching $v_{T}^{S F}$. Therefore, no CDP 478 is observed in such flows.

\section{Conclusion}

481 The present study aimed at proposing a methodology for the analysis of separating 482 open-channel flows. Works from the literature reported that for configurations with 483 large angles of separating streamline (referred to as "Group 1"), the analysis using a 484 Cartesian frame-axis leads to a change of sign of the Reynolds shear stress along with a 485 negative eddy viscosity coefficient. These results were retrieved in the lateral branch of 486 the present open-channel bifurcation which may represent a bifurcation in a river 487 network (in absence of sediments), an irrigation network, a sewer network or a 488 crossroad during urban flooding.

489 Moreover, we showed that such analysis in Cartesian frame-axis does not permit to 490 draw conclusions of size, shape and turbulent characteristics of the mixing layer. 491 Oppositely, we showed that using a so-called local "Serret-Frenet" frame-axis based on 492 the direction of the local mean velocity, the mixing layer data become explicit. The 493 shape, size and turbulent characteristics of the mixing layer are then similar to that of 494 the literature. This approach applies to any separating flow including the lateral channel 495 of open channel bifurcations of any angle and any channel width.

496 Finally, the eddy viscosity concept nicely applies in the local frame-axis, given the high 497 similarity between the Reynolds shear stress and the mean shear rate. Such local frame498 axis thus appears to be well-suited for characterizing the mixing layer of the separating 499 flow.

500 To conclude, we provide a generalization of the classical analysis of simple 2D mixing 501 layers (either straight or curved) reported in most textbooks for more complex 2D 502 mixing layers (among which the separating flows) using a simple and intuitive 503 approach. The use of this approach should be generalized to all mixing layers as long as 504 the main axis of the mixing layer deflects from the main direction of the axis system. 
505 The present results have major relevance to passive or reactive scalar (such as 506 pollutants) exchanges between the main flow and the recirculation zone. Light suspended sediments or dilute suspensions that follow the carrier flow, may be trapped in the recirculation. Therefore, locally, very high sediment concentrations may appear leading to deposition or agglomeration.

\section{Acknowledgment}

The research was funded by the INSA-Lyon BQR Program, the French INSU EC2COCytrix 2011 project No 231 and the French ANR-11-ECOTECH-007 project Mentor.

\section{References}

Adams, E.W., and J.P. Johnston (1988), Effects of the separating shear layer on the reattachment flow structure. Part 2: Reattachment length and wall shear stress, Exp. in Fluids 6, 493-499.

Babarutsi, S., J. Ganoulis, and V.H Chu (1989), Experimental investigation of shallow recirculating flows, J. Hydr. Engin. 115 (7), 906-924.

Bell, J., and R. Mehta (1990), Development of a two-stream mixing layer from tripped and untripped boundary layers, AIAA Journal, 28(12), 2034-2042.

Best, J. L., and I. Reid (1984), Separation zone at open-channel junctions, J. of Hydr. Engin., 110(11), 1588-1594.

Blanckaert, K., A. Duarte, Q. Chen, and A.J. Schleiss (2013), Near-bank processes in curved open-channel reaches: flow separation at the convex inner bank and reversed secondary flow at the concave outer bank, Submitted to J. of Geophys. Res.

Chu, V.H., F. Liu, and W. Altai (2004), Friction and confinement effects on a shallow recirculating flow, J. Environ. Engin. Sci. 3, 463-475.

Durbin, P.A. (1993), A Reynolds stress model for near wall turbulence, J. of Fluid Mech., 249, 465-498.

Fiedler, H., J.-H. Kim, and N. Köpp (1991), The spatially accelerated mixing layer in a tailored pressure gradient, Eur. J. Mech. B, 10(4), 349-376.

Gibson, M., and B. Younis (1983), Turbulence measurements in a developing mixing layer with mild destabilizing curvature, Exp. in Fluids, 1, 23-30.

Gurram, S.K., K.S. Karki, and W.H. Hager (1997), Subcritical junction flows, J. of Hydr. Engin., 123(5): pp 447-455.

Hattori, H., and Y. Nagano (2010), Investigation of turbulent boundary layer over forwardfacing step via direct numerical simulation, Int. J. of Heat and Fluid Flow, 31, 284-294.

Kasthuri, B., and N.V. Pundarikanthan (1987), Discussion of 'separation zone at open channel junction', J. of hydr. Engin., 113(4), 543-544.

Kuethe, A.M. (1935), Investigations of the turbulent mixing regions formed by jets, J. Appl. Mech., 2 (3), A87-A95.

Largeau, J.F., and V. Moriniere (2007), Wall pressure fluctuations and topology in separated flows over a forward-facing step, Exp. in Fluids, 42, 21-40.

Li, X. and Djilali N. (1995), On the scaling of separation bubbles, JSME Int. J. Series B, 38 (4), 541-548.

Loucks, R.B., and J.M. Wallace (2012), Velocity and velocity gradient based properties of a turbulent plane mixing layer, J. Fluid. Mech. 699, 280-319.

Margolis, D., and J. Lumley (1965), Curved turbulent mixing layer, Phys. of Fluids, 8(10), 1775-1784. 
Mignot, E., I. Vinkovic, D. Doppler, and N. Riviere (2013), Mixing layer in open-channel junction flows, Envir. Fluid Mech, DOI 10.1007/s10652-013-9310-7.

Neary, V., F. Sotiropoulos and A. Odgaard (1999), Three-dimensional numerical model of lateral-intake inflows, J. Hydr. Eng., 125(2), 126-140.

Paranthoen, P., G. Godard, F. Weiss, and Gonzalez M. (2004), Counter gradient diffusion vs "counter diffusion" temperature profile?, Int. of Heat and Mass Transfer, 47, 819-825.

Patel, V.C., and F. Sotiropoulos (1997), Longitudinal curvature effects in turbulent boundary layers. Prog. Aerospace Sciences, 33, 1-70.

Pope, S. B., (2000), Turbulent Flows. Cambridge University Press.

Plesniak, M., R. Mehta, and J. Johnston, (1996), Curved two-stream turbulent mixing layers revisited. Exp. Thermal and Fluid Science, 13, 190-205.

Ren, H., and Y. Wu (2011), Turbulent boundary layers over smooth and rough forward-facing steps, Phys. of Fluids 23, 045102.

Riviere, N, S. Gautier, and E. Mignot (2011a), Experimental characterization of flow reattachment downstream open channel expansions. 34th IAHR Congress, 26 June to 1st July 2011, Brisbane, Australia.

Rivière, N., G. Travin, and R. J. Perkins (2011b), Subcritical open channel flows in four branch intersections, Water Resour. Res., 47, W10517.

Rodi, W. (1993), Turbulence models and their application in Hydraulics - A state of the art review, IAHR, Delft, The Netherlands, $3^{\text {rd }}$ edition.

Sherry, M., D. Lo Jacono, and J. Sheridan (2010), An experimental investigation of the recirculation zone formed downstream of a forward facing step, J. Wind Engin. Ind. Aerodyn. 98, 888-894.

Simoens, S., M. Ayrault, and J.M. Wallace (2007), The flow across a street canyon of variable width - Part 1: Kinematic description, Atmos. Env., 41, 9002-9017.

Uijttewaal, W.S. and Booij, R. (2000), Effects of shallowness on the development of freesurface mixing layers, Phys. of Fluids, 12 (2), 392-402.

Van Prooijen, B. and W. Uijttewaal (2002), A linear approach for the evolution of coherent structures in shallow mixing layers, Phys. of Fluids, 14 (12), $4105-4114$.

Wygnanski, I., and H. Fiedler (1970), The two-dimensional mixing region, J. Fluid Mech., 41(2), 327-361.

\section{Appendix}

This appendix details the steps from Eq. (11) to Eq. (16). Applying Eq. (11) to the nondiagonal term of the Reynolds tensor in Cartesian frame reads:

$$
\mathbf{R}_{x y}=-\overline{u^{\prime} v^{\prime}}=\left(\overline{u_{s}^{\prime} u_{n}^{\prime}}\right) \cos (2 \theta)+\frac{\sin (2 \theta)}{2}\left(\overline{u_{n}^{\prime 2}}-\overline{u_{s}^{\prime 2}}\right)
$$

Now, applying Eq. 11 to the non-diagonal term of the mean shear rate in Cartesian frame leads to:

$\mathbf{S}_{x y}=\frac{1}{2}\left(\frac{\partial \bar{u}}{\partial x}+\frac{\partial \bar{v}}{\partial y}\right)=\frac{1}{2}\left\{\left(\frac{\partial \overline{u_{s}}}{\partial s}-\frac{\overline{u_{s}}}{R_{n}}\right) \sin (2 \theta)-\left(\frac{\partial \overline{u_{s}}}{\partial n}+\frac{\overline{u_{s}}}{R_{s}}\right) \cos (2 \theta)\right\}$

Thus, applying the eddy viscosity concept in Cartesian frame-axis $\left(\mathbf{R}_{x y}=v_{T}^{C} \cdot 2 \mathbf{S}_{x y}\right.$ in Eq.

2) using Eqs. 12 and 13 reads:

$v_{T}^{C}\left\{\left(\frac{\partial \overline{u_{s}}}{\partial s}-\frac{\overline{u_{s}}}{R_{n}}\right) \sin (2 \theta)-\left(\frac{\partial \overline{u_{s}}}{\partial n}+\frac{\overline{u_{s}}}{R_{s}}\right) \cos (2 \theta)\right\}=\left(\overline{u_{s}^{\prime} u_{n}^{\prime}}\right) \cos (2 \theta)+\frac{\sin (2 \theta)}{2}\left(\overline{u_{n}^{\prime 2}}-\overline{u_{s}^{\prime 2}}\right)$ 
594 and including the eddy viscosity concept in Serret-Frenet frame-axis (Eq. 6) in the first 595 Right Hand Side term of Eq. (14) gives:

596

$$
v_{T}^{C}\left\{\left(\frac{\partial \overline{u_{s}}}{\partial s}-\frac{\overline{u_{s}}}{R_{n}}\right) \sin (2 \theta)-\left(\frac{\partial \overline{u_{s}}}{\partial n}+\frac{\overline{u_{s}}}{R_{s}}\right) \cos (2 \theta)\right\}=-v_{T}^{S F}\left(\frac{\partial \overline{u_{s}}}{\partial n}+\frac{\overline{u_{s}}}{R_{s}}\right) \cos (2 \theta)+\frac{\sin (2 \theta)}{2}\left(\overline{u_{n}^{\prime 2}}-\overline{u_{s}^{\prime 2}}\right)
$$

597 that is

598

$$
v_{T}^{C}=\frac{-v_{T}^{S F}\left(\frac{\partial \overline{u_{s}}}{\partial n}+\frac{\overline{u_{s}}}{R_{s}}\right)+\tan (2 \theta)\left(\overline{u_{n}^{\prime 2}}-\overline{u_{s}^{\prime 2}}\right) / 2}{-\left(\frac{\partial \overline{u_{s}}}{\partial n}+\frac{\overline{u_{s}}}{R_{s}}\right)+\tan (2 \theta)\left(\frac{\partial \overline{u_{s}}}{\partial s}-\frac{\overline{u_{s}}}{R_{n}}\right)} .
$$

599 
Fig. 1. Sketch of the separation zone for one case of each group: the upward facing-step for group 1 and the downward-facing step for group 2; "Ss" stands for the mean flow axis at the geometrical singularity.

604 Fig. 2. Sketch of the experimental set-up and close- top photography view of the intersection zone, white dye has been injected in the main branch to visualize the mixing-layer (not used for quantitative data).

Fig. 3. Time-averaged velocity field along with separation streamline (plain line). Right graph is a velocity magnification to unveil the recirculation region.

611 Fig. 4. Distribution of mean shear rate $(+)$ along with Reynolds shear stress $(\diamond)$. The CDP zones

612 are indicated by shading and the plain thick line is the separating streamline.

614 Fig. 5. Application of eddy viscosity concept in Cartesian frame-axis. a: 2D distribution of

615 horizontal Reynolds shear stress. b: 2D distribution of $2 v_{T}^{C} S_{x y}$, with a constant eddy viscosity 616 value: $v_{T}^{C}=8.10^{-5} \mathrm{~m}^{2} / \mathrm{s}$. The plain line is the separating streamline.

618 Fig. 6. a: streamlines, separation streamline (thick line), and fieldlines (perpendicular to the 619 flow). b: velocity field with the Serret-Frenet frame-axis, the separating streamline and 7 620 fieldlines (plain grey lines) selected for analysis.

622 Fig. 7. Profiles of mean streamwise velocity $\left(\bar{u}_{s}\right.$, a), normal gradient of mean streamwise velocity $\left(\partial \overline{u_{s}} / \partial n, \mathrm{~b}\right)$ and Reynolds shear stress $\left(-\overline{u_{s}^{\prime} u_{n}^{\prime}}, \mathrm{c}\right)$ along the 7 fieldlines of Fig. $6 \mathrm{~b}$.

624 For sake of simplicity, orientation of the vertical axis was reversed.

Fig. 8. a: outer velocities $\mathrm{U1}(\mathrm{S})(+)$ and $\mathrm{U} 2(\mathrm{~S})(\mathrm{o})$, b: velocity scale and c: mixing layer width.

628 Fig. 9. Non-dimensional mean streamwise velocity profiles along the seven fieldlines of Fig. 6 b. For sake of simplicity, orientation of the vertical axis was reversed.

631 Fig. 10. Application of eddy viscosity concept in Serret-Frenet frame-axis a: 2D distribution of 632 horizontal Reynolds shear stress. b: 2D distribution of $2 v_{T}^{S F} S_{s n}$ with a constant eddy viscosity 633 value: $v_{T}^{S F}=5 \cdot 10^{-5} \mathrm{~m}^{2} / \mathrm{s}$. The white line is the separating streamline. 
635 Fig. 11. Evolution along S of eddy- viscosity coefficients $v_{T-\text { Best Fit }}^{S F}(\mathrm{o})$ and $v_{T-\text { Prandtl }}^{\mathrm{SF}}(+)$.

636

637 Fig. 12. Reynolds shear stress $-\overline{u_{s}^{\prime} u_{n}^{\prime}}$ (X) and application of Eq. (6) using: ( $)$

$638 v_{T-\text { Best Fit }}^{S F}\left(\partial \overline{u_{s}} / \partial n+\overline{u_{s}} / R_{s}\right)$, (o) the simplified version of Eq (6) $v_{T-\text { BestFit }}^{S F}\left(\partial \overline{u_{s}} / \partial n\right)$, and (घ)

$639 \quad v_{T-\text { Prandtl }}^{\mathrm{SF}}\left(\partial \overline{u_{s}} / \partial n+\overline{u_{s}} / R_{s}\right)$.

640

641 Fig. 13. Scheme of the Cartesian (x,y) and Serret-Frenet $(\mathrm{s}, \mathrm{n})$ frame-axes

642 